\title{
POTENTIAL-DENSITY BASIS SETS \\ FOR THREE-DIMENSIONAL DISKS
}

\author{
DAVID J.D. EARN \\ Racah Institute of Physics \\ The Hebrew University, Jerusalem 91904, Israel
}

Poisson's equation, $\nabla^{2} \psi=4 \pi G \rho$, can be solved approximately using basis sets of potential-density pairs. A given density is approximated by a truncated expansion; the computed expansion coefficients immediately yield the corresponding potential since each basis density function is paired with a basis potential function, and Poisson's equation is linear.

This idea leads to a powerful $N$-body simulation technique (CluttonBrock 1972) and is fundamental to semi-analytical normal mode analyses of stellar systems (Kalnajs 1977). The first step required before implementing these methods is to find a basis set that is well-suited to the model of interest. Some new basis sets for three-dimensional disks are presented here.

The standard potential-density basis set in cylindrical polar coordinates is given by the Laplacian eigenfunctions, $\psi_{k m h}(r, \phi, z)=J_{m}(k r) e^{i m \phi} e^{i h z}$, which are obtained by the method of separation of variables. $J_{m}$ is the cylindrical Bessel function of order $m$ where $m$ is an integer, and $k$ and $h$ range over all real values. The associated density function is $\rho_{k m h}(r, \phi, z)=$ $-\frac{k^{2}+h^{2}}{4 \pi G} \psi_{k m h}(r, \phi, z)$. These form a complete, orthogonal set for the space of square-integrable functions, $L^{2}\left(\mathbb{R}^{3}\right)$, but they are not especially well suited for numerical work with density distributions that are nearly flat, like disk galaxies. At the very least we want $\rho_{k m h}$ to decay as $z \rightarrow \pm \infty$.

More suitable basis sets can be derived by seeking separated potential and density functions that are not necessarily eigenfunctions of the Laplacian operator. Instead, we can specify the vertical density profile $\rho_{z}(z)$ in advance and seek solutions of Poisson's equation with $\psi(r, \phi, z)=$ $R(r) \Phi(\phi) Z(z)$ and $\rho(r, \phi, z)=\frac{1}{4 \pi G} R(r) \Phi(\phi) \rho_{z}(z)$. Then $R(r)=J_{m}(k r)$, $\Phi(\phi)=e^{i m \phi}$ and $Z(z)$ is the solution of

$$
\frac{d^{2} Z}{d z^{2}}-k^{2} Z=\rho_{z}(z)
$$


where we impose the boundary conditions that the potential factor $Z(z) \rightarrow$ 0 as $z \rightarrow \pm \infty$. The first row of Table 1 gives Green's function for Eq. (1) and yields the standard Bessel function basis set for flat disks. Rows 2-5 give solutions of Eq. (1) that allow us to construct 3-D basis sets for realistic disk galaxy models. To form basis sets we observe that $\nabla^{2}$ is invariant under the translation $z \rightarrow(z-h)$ for any $h$, so if we replace $Z(z)$ by $Z(z-h)$ and $\rho_{z}(z)$ by $\rho_{z}(z-h)$ then we have another potential-density pair. As will be shown elsewhere, if we take $\rho_{z}(z)$ from any row in Table 1 and let $h$ vary from $-\infty$ to $\infty$ then the density functions, $\rho_{k m h}(r, \phi, z)=\frac{1}{4 \pi G} J_{m}(k r) e^{i m \phi} \rho_{z}(z-h)$, form an $L^{2}$-complete basis.

TABLE 1. Vertical Factors for New Basis Sets

\begin{tabular}{lll}
\hline & Density factor $\rho_{z}(z)$ & Potential factor $Z(z)$ \\
\hline 1 & $k^{2} \delta(k z)$ & $-e^{-k|z|}$ \\
2 & $k^{2} e^{-k|z|}$ & $-\frac{1}{2}(1+k|z|) e^{-k|z|}$ \\
3 & $k^{2} \operatorname{sech}(k z)$ & $k z e^{k z}-\cosh (k z) \log \left(1+e^{2 k z}\right)$ \\
4 & $k^{2} \operatorname{sech}^{2}(k z)$ & $1+\sinh (k z) \arctan [\sinh (k z)]-\frac{\pi}{2} \cosh (k z)$ \\
5 & $k^{2} e^{-(k z)^{2}}$ & $-\frac{1}{4} e^{1 / 4} \sqrt{\pi}\left[e^{k z} \operatorname{erfc}(1 / 2+k z)+e^{-k z} \operatorname{erfc}(1 / 2-k z)\right]$ \\
\hline
\end{tabular}

Unlike the standard basis, the sets presented here are not biorthogonal. However, as emphasized by Saha (1993), the only extra effort required as a result is the one-time evaluation and inversion of a moderately large matrix. Since two of the three components of our basis functions are biorthogonal, the matrix involved is sparse off-diagonal and its computation and inversion always amounts to a negligible overhead. Note that since $k$ and $h$ are continuous indices (unlike $m$ ) the radial and vertical functions are selected by specifying $\Delta k, k_{\max }, \Delta h$ and $h_{\max }$ rather than simply the number of functions.

These basis sets are easy to implement: Bessel functions are available in all standard numerical libraries and the remaining factors are simple. They should, therefore, be very useful for stability studies and modeling of disk galaxies.

Acknowledgements. I am grateful to Ed Doolittle and Alar Toomre for helpful discussions. This research was supported by a Lady Davis postdoctoral fellowship.

\section{References}

Clutton-Brock, M. (1972) Astrophys. and Space Sci., 16, 101-119

Kalnajs, A.J. (1977) Astrophys. J., 212, 637-644

Saha P. (1993) Mon. Not. R. astr. Soc., 262, 1062-1064 